\title{
FORMACIÓN DE CÁLCULOS RENALES DE OXALATO CÁLCICO EN MAMÍFEROS
}

\section{FORMATION OF KIDNEY STONES OF CALCIUM OXALATE IN MAMMALS}

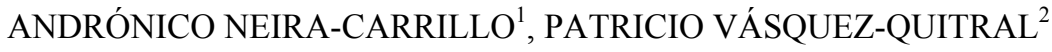

\begin{abstract}
Calcium oxalate $(\mathrm{CaOx})$ is the main inorganic component of urolithiasis and is mainly present in the formation of kidney stones in humans. Additionally, oxalocalcic urolithiasis has become the most frequent type of kidney stones, for instance, oxalocalcic urolithiasis has substantially increased in dogs and cats over the last decades. In urolithiasis, urine is supersaturated with calcium and oxalate ions that promote nucleation, growth and aggregation of $\mathrm{CaOx}$ crystals. It is accepted that the presence of acidic macromolecules and inorganic compounds in urine is responsible not only for the molecular processes involved in lithiasis but also for the adhesion and endocytosis by renal epithelial cells. Patients with the tendency to form kidney stones may have an alteration in the structure and/or function of some inhibitors of crystallization. In the current review, we describe some general aspects of epidemiology and pathophysiology of oxalocalcic urolithiasis.
\end{abstract}

KEY WORDS: Calcium oxalate, kidney stones, oxalocalcic urolithiasis, crystallization.

\section{INTRODUCCIÓN}

El contenido mineral constituye la fracción mayoritaria (alrededor 95\% del peso) de los urolitos o cálculos en las vías urinarias. La litiasis urinaria corresponden a concreciones constituidas por sales urinarias, las cuales generalmente están formados casi exclusivamente por urolitos de oxalato cálcico $(\mathrm{CaOx})$, aunque algunos contienen cantidades variables de fosfato cálcico, o una mezcla de ambos (Chew et al. 1998; Tomaszewski et al., 2001). En la naturaleza, el $\mathrm{CaOx}$ posee tres polimorfos cristalinos: monohidratado (COM), dihidratado (COD) y trihidratado (COT) (Monje y Baran, 2002). El cristal $\mathrm{COM}$ es el polimorfo más predominante de la matriz orgánica en urolitos de $\mathrm{CaOx}$ de humanos, perros y gatos. La morfología de los cristales COM corresponden a cristales hexagonales alargados, por su parte los cristales COD presentan una forma de

${ }^{1}$ Laboratorio de Materiales Bio-relacionados, Departamento de Ciencias Biológicas y Animales,

Facultad de Ciencias Veterinarias y Pecuarias, Universidad de Chile. aneira@uchile.cl

${ }^{2}$ Doctorando en Ciencias Silvoagropecuarias y Veterinarias, Campus Sur, Universidad de Chile 
bipirámide de base cuadrada. La Figura 1 muestra esquemáticamente las fases cristalográficas de $\mathrm{COM}$ y de COD (Fig. 1A,B) e imágenes de microscopía electrónica de barrido (SEM) de ambos polimorfos (Fig. 1C,D) obtenidos in vitro con un nuevo polidimetilsiloxano sintetizado en el laboratorio de Materiales Bio-relacionados de nuestra facultad.

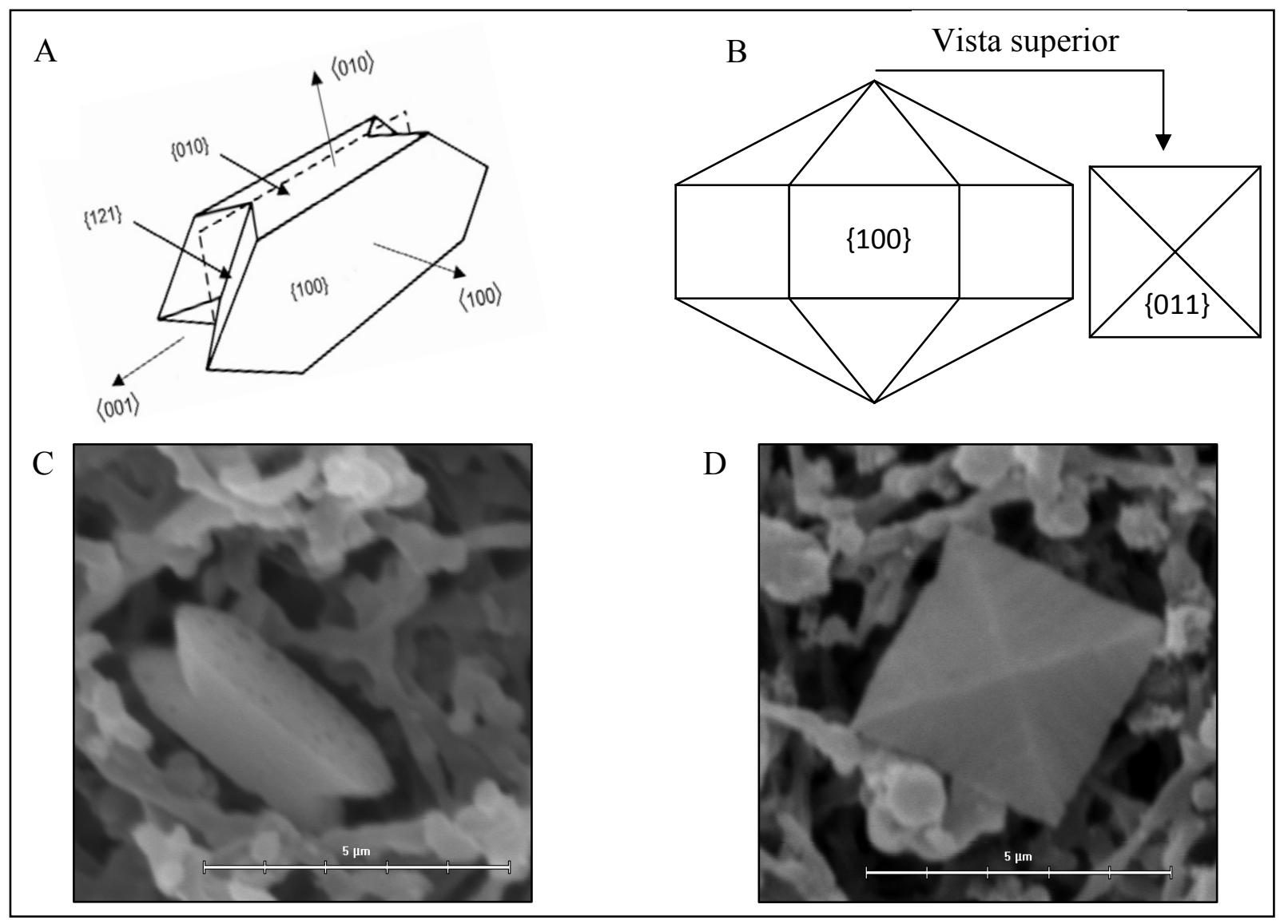

Figura 1. Fases cristalográficas $(\mathrm{A}, \mathrm{B})$ y SEM de cristales de CaOx estabilizados in vitro a $\mathrm{pH} 9$ por 24 horas usando un nuevo polidimetilsiloxano sintetizado en el Laboratorio de Materiales Bio-relacionados de nuestra facultad. A) Oxalato de calcio monohidratado (COM) y B) Oxalato de calcio dihidratado (COD), C) SEM de un cristal COM y D) SEM de un cristal COD.

Se ha reportado que la urolitiasis canina presenta ambos polimorfos: COM y COD, los cuales están constituidos en distintas capas del mismo urolito (Buffington et al., 1997). Un aspecto clínico relevante entre estos polimorfos es que los urolitos formados de cristales COD se fragmentan con mayor facilidad mediante ondas de choque que los urolitos constituidos por COM (Byrne et al., 1999). Por otro lado, los cristales COT no tienes importancia clínica ya que es termodinámicamente muy inestable, resultando muy poco probable encontrarlo en urolitos de $\mathrm{CaOx}$. Es importante destacar que la prevalencia de la urolitiasis por $\mathrm{CaOx}$ aumenta anualmente tanto en personas como en animales de compañía pese al 
desarrollo de métodos analíticos cada vez más eficientes para la determinación de marcadores litógenos, técnicas de diagnóstico clínico y tratamientos menos invasivos fundamentalmente con el manejo dietario.

\section{EPIDEMIOLOGÍA}

Los cálculos urinarios afectan a 10-12\% de la población en países industrializados (Bihl y Meyers, 2001). Su incidencia ha estado en aumento los últimos años, a su vez la edad de inicio está disminuyendo (Finlayson, 1974). Adicionalmente, la recurrencia es alta siendo superior a 50\% (Wassertein, 1998). La urolitiasis es un desorden común en perros en todas las áreas geográficas y el diagnóstico de perros con enfermedad del tracto urinario bajo puede alcanzar hasta un $18 \%$ (Lulich et al., 2000). Por otro lado, el porcentaje de urolitos caninos de $\mathrm{CaOx}$ estudiados en el Minnesota Urolith Center ha aumentado desde un $5 \%$ en 1981 hasta un $35 \%$ en 1997, convirtiéndose en los segundos urolitos más habituales en los perros (Buffington et al., 1997). Algunas investigaciones respecto de la prevalencia de urolitos de $\mathrm{CaOx}$ en animales pequeños señalan al ion magnesio $(\mathrm{Mg})$ como una potencial inductor de la urolitias urinaria. Por tal razón, fabricantes de alimentos han restringido el contenido de $\mathrm{Mg}$ en las dietas y han agregado ingredientes para aumentar la acidez de la orina en los alimentos. Los acidificantes urinarios aumentan la solubilidad de urolitos, por ejemplo, de los cristales de estruvita en gatos favoreciendo con esto la liberación de carbonato cálcico del hueso produciendo de esta manera hipercalciuria (Dibartola, 2000).

Se ha descrito una alta recurrencia de la urolitiasis canina. Siendo los urolitos de $\mathrm{CaOx}$ más frecuentes en los perros macho (del 68\% al 71\%) que en las perras (del 26\% al 32\%) (Marchand et al., 1998). Esta misma tendencia se observa en humanos, en la cual los varones desarrollan tres veces más urolitiasis que las mujeres. Esta tendencia podría ser explicada por una disminución de la excreción urinaria del oxalato (inducida por los estrógenos) y a la excreción excesiva de citrato en la orina de las hembras (Lund et al., 1999). Se ha comprobado que los gatos machos tienen 1.5 veces más probabilidades de desarrollar urolitos de $\mathrm{CaOx}$ y los gatos castrados presentan siete veces más probabilidades que los sexualmente intactos. Por otro lado, la obesidad es una condición muy importante a considerar, se ha visto que los perros obesos existe mayor probabilidad que desarrollen urolitos de $\mathrm{CaOx}$ al igual que los gatos y los seres humanos con sobrepeso (Lund et al., 1999; Rupniak y Kramer, 1999). Es conocido que el pH urinario acídico de personas con sobrepeso aumenta el riesgo de formación de cálculos renales.

La tendencia a la formación de litiasis urinaria también la presentan las diferentes razas tanto de perros como de gatos. Las razas de perros con mayor riesgo a formar urolitos de $\mathrm{CaOx}$ son: Schnauzer Miniatura, Lhasa Apso, Yorkshire Terrier, Bichon Frise, Pomerania, Shih Tzu, Cairn Terrier, Maltés, Caniche Miniatura y Chihuahua (Hill, 2000). Entre las razas de gatos con mayor riesgo a la urolitiasis se encuentran: Ragdoll, Gatos Británicos Pelo Corto, Foráneos de Pelo Corto, Himalayas, Havana Brown, Scottish Folds, Persas y Exóticos de Pelo Corto.

\section{ETIOPATOGENIA}

Sin lugar en la patogenia de la litiasis urinaria intervienen diferentes aspectos mecanísticos, tales como la saturación salina de la orina, la poca eficacia de los inhibidores de la cristalización, la formación de núcleos de tamaños críticos que favorecen las etapas 
de inducción de cristales, reducción del volumen urinario, infecciones, etc. A continuación se describen algunos aspectos de la composición de la orina, que son relevantes en la formación de cálculos renales en general:

- Sobresaturación. Cuando una solución contiene un soluto cristalizable en cantidad superior a la solubilidad permitida por su producto de solubilidad (Kps) se dice que el sistema está sobresaturado. Por lo tanto, sobre ciertos valores de saturación (sobresaturación crítica) se produce la precipitación urinaria en forma instantánea. El intervalo entre la concentración de saturación de un soluto y la concentración a partir de la cual éste precipita instantáneamente se denomina zona metaestable. En esta zona, la solución urinaria se encuentra en un estado termodinámicamente inestable, en el cual los solutos de la solución urinaria comienzan a cristalizar con el fin de lograr un de equilibrio termodinámico. En la mayoría de los vertebrados la orina se encuentra permanentemente sobresaturada respecto a $\mathrm{CaOx}$ (Finlayson, 1978). Por su parte, el valor del pH urinario también produce que la orina se encuentre sobresaturada, por ejemplo, un $\mathrm{pH} 5.5$ provoca una sobresaturación respecto al ácido úrico y un valor de pH 6.0 la cristalización del fosfato cálcico en forma de hidroxiapatita (Grases, 1997).

-Déficit de inhibidores de la cristalización. En la orina existen sustancias orgánicas e inorgánicas, las cuales actúan como factores inhibidores de la cristalización. Estos inhibidores son capaces de evitar o dificultar la formación de cristales de distinta composición química y estructura (Lieske, 1996). Esta es la principal razón por la cual gran parte de la población no desarrolla cálculos renales. La efectividad de estos factores de inhibición depende de su concentración y de la sobresaturación de sus solutos cristalizables, siendo cruciales en los casos en que no se alcanzan sobresaturaciones críticas demasiado elevadas en la orina.

- Aumento de los factores promotores de la cristalización. La precipitación de productos cristalinos insolubles o nucleantes heterogéneos en la orina pueden quedar atrapadas en las vías urinarias constituyendo núcleos críticos sobre los cuales se produce el crecimiento del cálculo. En la litiasis urinaria, los cristales de ácido úrico (a $\mathrm{pH}$ inferiores a 5.5) y de fosfato cálcico (a $\mathrm{pH}$ superiores a 6.0), la presencia de compuestos orgánicos tales como: macromoléculas, restos celulares y lesiones epiteliares, los fármacos $\mathrm{y}$ sus metabolitos son nucleantes heterogéneos a considerar (Grases 1992).

Es importante considerar que generalmente los factores promotores de la cristalización están compensados por los factores inhibidores de la cristalización, evitando así la formación de cristales de $\mathrm{CaOx}$. Sin embargo, cuando se produce un desbalance ya sea en la disminución de la síntesis y/o secreción de inhibidores, o un aumento no compensado de factores promotores de cristalización ocurre un proceso de cristalización o mineralización patológica, conocido como calculogénesis oxalocálcica. Adimás, se describen otras condiciones que predisponen a la formación de urolitos de $\mathrm{CaOx}$, entre las cuales podemos mencionar:

- Hipercalciuria. El aumento de la excreción urinaria de calcio (hipercalciuria) es un factor común que contribuye a la urolitiasis de $\mathrm{CaOx}$ en los seres humanos, gatos y perros (Welk y Buffington, 2003; de Groat et al., 1993; Sands et al., 2000). Se ha propuesto que la hipercalciuria surge por uno de estos tres mecanismos: a) hipercalciuria por absorción, b) hipercalciuria por pérdida renal, c) hipercalciuria por resorción.

La hipercalciuria por absorción en seres humanos y perros se caracteriza por un aumento de la 
absorción intestinal de calcio y un exceso de la excreción de calcio en la orina (Lund et al., 1999). Un aumento leve del calcio sérico, como consecuencia de una mayor absorción intestinal de calcio, produce una mayor carga de filtrado de éste a nivel glomerular y disminuye su reabsorción tubular, al mismo tiempo su alta concentración sérica suprimiría la liberación de hormona paratiroidea (PTH). La combinación del aumento del calcio filtrado y la disminución de la reabsorción tubular provoca hipercalciuria. El aumento de absorción intestinal en el yeyuno se acompaña de un aumento en la absorción de oxalato lo que contribuye a la co-existencia de una hiperoxaluria Se ha descrito que un $50 \%$ de las personas con hipercalciuria por absorción intestinal tienen un aumento de 1,25-dihidroxicolecalciferol (DHCC) incrementando la absorción intestinal de calcio (Valentino et al., 1999). Un estudio realizado en perros de raza Schnauzer indicó que cinco de seis Schnauzer miniatura con urolitiasis de $\mathrm{CaOx}$ presentaron hipercalciuria por absorción (Welk y Buffington, 2003). La hipercalciuria por pérdida renal se debe a un trastorno de la absorción tubular renal de calcio (Lund et al., 1999). La pérdida de calcio renal disminuye el calcio sérico, lo que produce un aumento de PTH y DHCC. El incremento de DHCC produce una mayor absorción intestinal de calcio y el incremento de la PTH hace que aumente la movilización del calcio desde el hueso. Los seres humanos y los perros con hipercalciuria por pérdida renal tienen concentraciones séricas de PTH altas, concentraciones séricas de calcio normales y una alta excreción urinaria de calcio durante el ayuno. En la hipercalciuria por resorción o resortiva hay niveles elevados de calcio en la orina (hipercalciuria hipercalcémica). Usualmente esta condición es resultado de enfermedades que cursan con resorción ósea excesiva, tales como hiperparatiroidismo primario y secundario, osteoporosis, etc.

-Hiperoxaluria. La concentración de oxalato $(\mathrm{Ox})$ en la orina tiene un mayor efecto sobre la saturación urinaria de $\mathrm{CaOx}$ que un aumento equivalente de calcio en la orina (Lund et al., 1999; Sands et al., 2000). La absorción de Ox ocurre a lo largo del intestino (Binder, 1974; Hatch y Freel, 2005) y colón, sin embargo este último absorbería una cantidad marginal de Ox (Balaji y Menon, 1997). Debido a que el Ox no es significativamente metabolizado en el organismo, su excreción comienza inmediatamente luego de su ingestión y alcanzando un máximo entre 2 a 6 horas (Prenen et al., 1984). Una vez absorbido el $\mathrm{Ox}$ es rápidamente captado, filtrado y eliminado por los riñones mediante filtración glomerular $\mathrm{y}$ eventualmente excretado en la orina (Holmes et al., 2005). El Ox urinario deriva principalmente de la producción endógena en el hígado procedente del metabolismo del ácido ascórbico (vitamina $\mathrm{C}$ ), glioxilato y glicina. A su vez, la ingestión de proteína derivada de la carne aporta los aminoácidos hidroxiprolina y triptófano que se metabolizan a $\mathrm{Ox}$ (Holmes y Assimos, 1998). La oxidación de los aminoácidos hidroxiprolina y glicina generan glioxilato, el cual es finalmente convertido a $\mathrm{Ox}$ a nivel hepático (Knight et al., 2006). La Figura 2 muestra el metabolismo de la absorción y excreción del Ox a nivel de la circulación sistémica y del tracto intestinal respectivamente, y su interacción con el hígado, órganos y tejidos estructurales. 


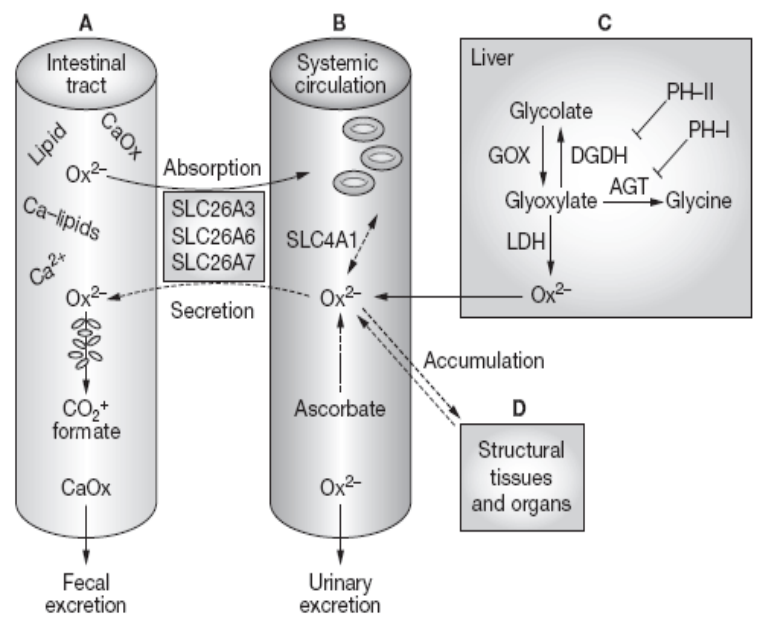

Figura 2. Metabolismo del oxalato

Adicionalmente, aminoácidos aromáticos como fenilalanina y tirosina pueden formar tautómeros enol inestables por desaminación lo que también genera Ox (Schwarz, 1961). Más del 50\% de los seres humanos con urolitiasis de $\mathrm{CaOx}$ tienen varios grados de hiperoxaluria (Lund et al., 1999), sin embargo en los perros con urolitiasis de $\mathrm{CaOx}$ no se ha detectado hiperoxaluria (Welk y Buffington, 2003). Se distinguen dos tipos de hiperoxaluria en personas: hiperoxaluria primaria $(\mathrm{PHO})$ y secundaria $(\mathrm{SHO})$. $\mathrm{La}$ PHO es un desorden metabólico raro asociado a herencia autosómica recesiva causado por déficit en las enzimas alanino-glioxilato aminotransferasa (AGT) (PHO tipo 1) y glioxilato reductasa (GR) (PHO tipo 2) (Cochat, 1999). Un déficit enzimático determina una conversión excesiva de glioxilato hacia Ox, que al ser poco soluble, es excretado en la orina causando nefrosis o urolitiasis de $\mathrm{CaOx}$ recurrente en seres humanos. Por su parte, en perros y gatos la oxaluria se asocia a nefrosis por Ox más que a urolitiasis de $\mathrm{CaOx}$ (Buffington, 1998; Stevens y Brenner, 1996). La SHO resulta de un aumento en la disponibilidad de sustrato como ácido ascórbico, etilenglicol y metoxifluorano (Conyers et al., 1990), hiperabsorción intestinal de Ox causada por dieta abundante en $\mathrm{Ox}$, deficiencia de vitamina $\mathrm{B}_{6}$, deficiente absorción de grasas, hiperoxaluria entérica o un desbalance entre $\mathrm{Ca}^{2+}$ y $\mathrm{Ox}$ intraluminal del intestino, por ejemplo debido a una dieta escasa en iones $\mathrm{Ca}^{2+}$ (Marangella et al., 1982; Curhan et al., 1993; Massey y Sutton, 1993; Takei et al., 1998; Nishiura et al., 2002).

Pese a los antecedentes anteriores una completa comprensión de las fuentes de Ox urinario es aún incompleta y el aporte relativo de la dieta es incierto en personas, más aun en animales de compañía debido a la escasez de estudios. Algunos autores reportan que la contribución de Ox de la dieta a la excreción urinaria es cercana al $10 \%$ en personas (Massey y Sutton, 1993; Von Unruh et al., 2003; Holmes et al. 2001), lo cual demuestra que la contribución de Ox dietario puede alcanzar el 50\%. Por su parte, la cantidad de Ox libre en el tracto gastrointestinal es afectada no sólo por la ingesta dietaria de Ox sino también por su unión a iones $\mathrm{Mg}^{2+}$, ácidos grasos, sales biliares e iones $\mathrm{Ca}^{2+}$ (Siener y Hess, 1995; Ferraz et al., 2004).

\section{- Modificadores de la cristalización de oxalato cálcico.}

La razón de que sólo algunas personas tengan urolitos radica en que la orina normalmente posee macromoléculas urinarias acídicas que inhiben no sólo la nucleación, agregación y/o crecimiento de urolitos (Hess et al., 1993) sino también la adhesión y endocitosis en células tubulares renales (Lieske et al., 2000; Tsujihata et al., 2006). Dos macromoléculas biológicas que inhibirían la formación de cálculos renales humanos $(\mathrm{CRH})$ son la Osteopontina (OPN) y la proteína Tamm-Horsfall (THP). La OPN es una glicoproteína fosforilada acídica aislada desde la matriz del hueso que contiene dominios ricos en ácido 
aspártico y ácido glutámico. La OPN modularía la mineralización normal del hueso al aumentar la actividad osteoclástica e inhibiendo el crecimiento de cristales de hidroxiapatita (Giachelli y Steiz, 2000). Es conocido que la OPN también es expresada por células epiteliales renales normales, particularmente células del segmento ascendente del asa de Henle y túbulos contorneados distales (Mazzali, 2002). Por otro lado, la THP también es expresada por células epiteliales renales pero a diferencia de OPN la THP es específica del riñón. La THP se sintetiza específicamente en la porción ascendente gruesa medular y en el túbulo distal (Serafini-Cessi, 2003). El mecanismo mediante el cual las proteínas OPN y THP ejercen su acción inhibitoria sobre la cristalización de $\mathrm{CaOx}$ in vivo está siendo intensamente investigado. Se postula que la unión directa entre estas biomoléculas y iones $\mathrm{Ca}^{2+} \mathrm{O}$ cristales-Ca ${ }^{2+}$ serían cruciales. Ambas proteínas contienen sitios ligantes de iones $\mathrm{Ca}^{2+}$, la OPN presenta uno mientras la THP posee dos (Mazzali et al., 2002). Complementariamente, OPN y THP pueden unir iones $\mathrm{Ca}^{2+}$ a través de sus grupos cargados negativamente, derivados de los ácidos siálico y fosfórico, respectivamente (Van Rooijen et al., 1999). Como consecuencia de estas reacciones de unión se produce una: reducción de la concentración de iones $\mathrm{Ca}^{2+}$ intratubular, inhibición de la agregación y crecimiento de cristales- $\mathrm{Ca}^{2+}$, y un bloqueo de la adhesión de cristales de $\mathrm{CaOx}$ a células epiteliales renales (Asselman y Verkoelen, 2002). Por lo tanto, si los inhibidores de la cristalización son defectuosos o escasos pueden contribuir a la formación de urolitos. La forma autoagregada de la THP, que es la forma predominante de esta proteína en la orina de los seres humanos con urolitos de $\mathrm{CaOx}$, es un inhibidor menos eficaz de la agregación de los cristales de $\mathrm{CaOx}$ e incluso puede facilitar la cristalización de
CaOx (Sugaya et al., 2002). La función de la THP en los perros con urolitos de $\mathrm{CaOx}$ es desconocida y en humanos la remoción enzimática del ácido siálico desde la THP disminuye parcialmente la inhibición del crecimiento y agregación de cristales de $\mathrm{CaOx}$ ejercida por THP nativa (Chen et al., 2001).

Por otro lado, en la orina también existen inhibidores inorgánicos de la calculogénesis oxalocálcica, entre ellos los más importantes son el citrato, magnesio y pirofosfato:

- Citrato. En la orina el citrato forma complejos con calcio que son más solubles que $\mathrm{CaOx}$, disminuyendo así la concentración de calcio iónico. El citrato inhibe la nucleación espontánea y la nucleación heterogénea de los cristales de $\mathrm{CaOx}$ (Lund et al., 1999). En los seres humanos el citrato se filtra por los riñones y aproximadamente el $75 \%$ se reabsorbe en los túbulos y el estado ácido-básico es el determinante de la cantidad que se reabsorbe. La disminución de citrato en la orina (hipocitraturia) es causada principalmente por la acidosis metabólica en seres humanos. La acidosis metabólica reduce la excreción urinaria de citrato al aumentar su reabsorción a nivel tubular. Por otro lado, la alcalosis metabólica disminuye la reabsorción tubular de citrato aumentando así su excreción urinaria (Pertovaara et al., 1991). En los perros la excreción urinaria de citrato es muy inferior a la de los seres humanos y menos del $1 \%$ filtrado se excreta normalmente en la orina, aunque la excreción es mayor en condiciones de alcalosis metabólica (Pertovaara et al., 1991). El citrato puede ser administrado en forma oral y parece ser un efectivo tratamiento contra la formación de CRH, el mecanismo potencial por el cual el citrato previene la formación de $\mathrm{CRH}$ incluye: aumento del $\mathrm{pH}$ urinario, disminución de la agregación de la proteína THP y disminución de la adhesión de cristales a células tubulares (Hess et al., 1993). 
- Magnesio. El magnesio al igual que la función del citrato con el calcio, se combina con el Ox reduciendo su saturación urinaria (Lund et al., 1999). Si la concentración urinaria de $\mathrm{Mg}$ es deficiente, hay más Ox disponible para combinarse con el calcio y por tanto una dieta baja en $\mathrm{Mg}$ predispone a la urolitiasis de $\mathrm{CaOx}$ (Caito, 1995; Monneron et al., 2000). - Pirofosfato. El Pi es otro inhibidor de la nucleación, crecimiento y agregación de cristales de $\mathrm{CaOx}$. El Pi corresponde a dos moléculas de fosfato unidas por un enlace éster. El Pi se une en forma irreversible a la superficie de los cristales de $\mathrm{CaOx}$. La cantidad de Pi en la orina se relaciona con la ingestión de fósforo en la dieta. Aunque no se conoce la función del Pi en la orina canina, se ha demostrado que dietas pobres en fósforo aumentan el riesgo de la urolitiasis de $\mathrm{CaOx}$ en los perros, lo cual estaría relacionado con una disminución de Pi en la orina (Caito, 1995; Monneron et al., 2000).

Recientemente, el uso de polímeros ha sido descrito como potenciales macromoléculas capaces de controlar selectivamente la cristalización in vitro de materiales inorgánicos tales como: oxalato de calcio (CaOx), carbonato de calcio $\left(\mathrm{CaCO}_{3}\right)$ (Wegner, 2000; Liu et al., 2003), y otros. Respecto de la estabilización selectiva de los polimorfos del $\mathrm{CaOx}$, polímeros a base del silicio conocidos como polidimetilsiloxano (PDMS) están siendo usados en nuestro laboratorio. Los PDMS son polímeros que presentan en su cadena principal enlaces silicio-oxígeno con grupos metilos sobre el átomo silicio. Los PDMS y sus derivados presentan diversas aplicaciones médicas y no médicas, debido a que estos polímeros presentan: altamente flexibilidad, permeabilidad a gases, biocompatibilidad, biodegradabilidad, son térmicamente estables y fácil de modificar químicamente, etc. (Neira-Carrillo et al., 2009). Sus aplicaciones en medicina han sido múltiples como por ejemplo: agente antiespumante en formulaciones farmacéuticas y alimentos, excipientes en dispositivos de liberación controlada, antiaglutinante en alimentos, lentes de contacto, sistemas de drenajes post-quirúrgico y para glaucoma, etc. Dado que la superficie de los PDMS puede ser modificada y funcionalizada quimicamente mediante la incorporación de grupos químicos aniónicos como: carboxílico, sulfónico, fosfórico similares a los que existen en macromoléculas acídicas urinarias que modulan la cristalización de $\mathrm{CaOx}$. En este contexto, el Laboratorio de Materiales Bio-relacionados de nuestra Facultad ha sintetizado, caracterizado y reportado un nuevo copolímero aniónico derivado de PDMS con grupos carboxílicos (Neira-Carrillo et al., 2010) como un eficiente modulador de la cristalización de $\mathrm{CaOx}$ in vitro. Este nuevo derivado de PDMS favorece la formación selectiva de cristales COD, el cristal no patológico presente en la personas que no desarrollan urolitos de $\mathrm{CaOx}$. ( ver Figura 1D)

\section{CONCLUSIONES}

El CaOx representa el principal componente de la urolitiasis oxalocálcica, la cual se encuentra presente mayoritariamente en la formación de $\mathrm{CRH}$ y su incidencia ha aumentado sustancialmente en perros y gatos en las últimas décadas. Dado que en la litiasis urinaria, la orina está sobresaturada de iones $\mathrm{Ca}^{2+} \mathrm{y}$ Ox, los cuales promueven la nucleación, crecimiento y agregación de cristales $\mathrm{CaOx}$; una evaluación cuantitativa de la cantidad de estos iones en orina y suero es muy recomendada en animales de compañía con urolitos de $\mathrm{CaOx}$ (Leumann y Hoppe, 2001; Milliner, 2005). Adicionalmente, se recomienda evitar el consumo de agua con alto contenido de calcio o pobre en minerales traza como el zinc, que se une al calcio, un monitoreo del DHCC en sangre y reducir la ingesta dietaria, en especial de alimentos 
ricos en oxalato (Stauffer, 1977). Creemos que una absorción intestinal y producción endógena disminuída de $\mathrm{Ox}$ podrían ser beneficiosas en invididuos con hiperoxaluria (Hess, 1996; Robertson y Peacock, 1980). Si bien ha existido un avance importante en torno al conocimiento de la formación de la urolitiasis de $\mathrm{CaOx}$ en las últimas décadas, aún persisten muchas interrogantes por resolver respecto a los mecanismos moleculares involucrados en la nucleación y estabilización de los polimorfos del $\mathrm{CaOx}$ en estos tipos de cálculos (Benítez y Talham, 2005).

\section{RECONOCIMIENTOS}

El Dr. Neira-Carrillo agradece el financiamiento aportado por el proyecto "Proyecto ENL 10/02, de la Vicerrectoría de Investigación y Desarrollo de la Universidad de Chile".

\section{REFERENCIAS}

ASSELMAN, M.; VERKOELEN, C. 2002. Crystal-cell interaction in the pathogenesis of kidney stone disease. Curr. Opin. Urol. 12: 271-276.

BALAJI, K. C.; MENON, M. 1997. Mechanism of stone formation. Urol. Clin. North Am. 24: 1-11.

BENITEZ, I.; TALHAM, D. 2005. Calcium oxalate monohydrate precipitation at membrane lipid raft. J. Am. Chem. Soc. 127: 2814-2815.

BIHL, G.; MEYERS, A. 2001. Recurrent renal stone disease-advances in pathogenesis and clinical managment. Lancet. 358: 651-656.

BINDER, H. J 1974. Intestinal oxalate absorption. Gastroenterology 67: 441-446.

BUFFINGTON, C. A. 1998. Functional assessment of $\alpha-2$ adrenoreceptor sensitivity in cats with interstitial cystitis. Soc. Neurosci. 24: 595.

BUFFINGTON, C. A.; CHEW, D. J.; KENDALL, M. S.; SERIVANI, P. V., THOMPSON, S.B.; BLAISDELL, J. L.; WOODWORTH, B. E. 1997. Clinical evaluation of cats with nonobstructive urinary tract diseases. J. Am. Vet. Med. Assoc. 210: 46-50.

BYRNE, D. S.; SEDOR, J. F.; ESTOJAK, J.; FITZPATRICK, K. J.; CHIURA, A. N.; MULHOLLAND, S. G. 1999. The urinary glycoprotein GP51 as a clinical marker for interstitial cystitis. J. Urol. 161: 1786-1790.

CAITO M. 1995. The presence and distribution of substance $\mathrm{P}$ and neuron-specific enolase in the feline bladder. En: Veterinary Anatomy. Columbus, Ohio State University, p 66.

CHEN, W. C.; LIN, H. S.; CHEN, H. Y.; SHIH, C. H.; LI, C. W. 2001. Effects of Tamm-Horsfall protein and albumin on calcium oxalate crystallization and importance of sialic acids. Molecular Urology 5: 1-5.

CHEW, D.J.; BUFFINGTON, C. A.; KENDALL, M. S.; DIBARTOLA, S. P.; WOODWORTH, B. E. 1998. Amitriptyline treatment for severe recurrent idiopathic cystitis in cats. J. Am. Vet. Med. Assoc. 213: 1282-1286.

Cochat, P. 1999. Primary hyperoxaluria type 1. Kidney Int. 55: 2533-2547.

CONYERS, R. A.; ROFE, A. M. 1990. The relation of clinical catastrophes, endogenous oxalate production, and urolithiasis. Clin. Chem. 36: 1717-1730.

Curhan, G.C.; Willett, W. C.; Rimm, E. B.; Stampfer, m. J. 1993. A prospective study of dietary calcium and other nutrients and the risk of symptomatic kidney stones. $\mathrm{N}$. Engl. J. Medicine 328: 833-838.

DE GROAT, W. C.; BOOTH, A. M.; YSHIMURA, N. 1993. Neurophysiology of micturition and its modification in animal models of human disease. In Nervous Control of the Urogenital System. The Autonomic Nervous System, Chur, Harwood, 1993, pp 227-290.

DIBARTOLA, S. P. 2000. Metabolic acid-base disorders. En: Dibartola, S. P. (ed). Fluid Therapy in Small Animal Practice. Philadelphia, WB Saunders Co., pp 211-240.

FERRAZ, R.R.; TISELIUS, H. G.; HEILBERG, I. P. 2004. Fat malabsorption induced by gastrointestinal inhibitor leads to an increase in urinary oxalate excretion. Kidney Int. 66: 676-682.

FINLAYSON, B. 1974. Symposium in renal lithiasis. Renal lithiasis in review. Urol. Clin. North Am. 1: 181-212. 
FinlaYson, B. 1978. Physicochemical aspects of urolithiasis. Kidney Int. 13: 344-360.

GIACHELLI, C.; STEIZ, S. 2000. Osteopontin: a versatile modulator of inflamation and biomineralization. Matrix Biol. 19: 615-622.

GRASES, F.; MILLÁN, A.; SÖHNEL, O. 1992. Role of agglomeration in calcium oxalate monohydrate urolith development. Nephron. 61: 145-150.

GRASES, F.; VILLACAMPA A. I.; SÖHNEL, O.; KÖNIGSBERGER, E.; MAY, P. M. 1997. Phosphate composition of precipitates from urine-like liquors. Cryst. Res. Technol. 32: 707-715.

HATCH, M.; FREEL, R. W. 2005. Intestinal transport of an obdurate anion. Urol. Res. 33: 1-18.

HESS, B.; Jost, C.; Zipperle, L.; Takkinen, R.; Jaeger P. 1998. High-calcium intake abolishes hyperoxaluria and reduces urinary cristallization during a 20-fold normal oxalate load in humans. Nephrol. Dial. Transplant. 13: 2241-2247.

HESS, B.; ZIPPERLE, L.; JAEGER, P. 1993. Citrate and calcium effects Tamm-Horsfall glycoprotein as a modifier of calcium oxalate crystal aggregation. Am. J. Physiol. 265: F784-F791.

HILL, R. 2000. Reply: will changing the testing paradigms show that NK1 receptor antagonists are analgesic in humans. Trends Pharmacol. Sci. 21:465.

HOLMES, R. P.; ASSIMOS, D. G. 1998. Glyoxylate synthesis, and its modulation and its influence on oxalate synthesis. J. Urol. 160: 1617-1624.

HOLMES, R. P.; GOODMAN, H. O.; ASSIMOS, D. G. 2005. Dietary oxalate loads and renal oxalate handling. J. Urol. 174: 943-947.

HOLMES, R.; GOODMAN, H.; ASSIMOS, D. 2001. Contribution of dietary oxalate to urinary oxalate excretion. Kidney International 59: 270-276.

HOPPE, B.; LEUMANN, E. 2001. Diagnostic and therapeutic strategies in hyperoxaluria: a plea for early intervention. Nephrol. Dial. Transplant. 19: 39-42.

KNIGHT, J.; JIANG, J.; ASSIMOS, D. G.; HOLMES, R. P. 2006. Hydroxyproline ingestion and urinary oxalate and glycolate excretion. Kidney Int. 70: 1929-1934.
LIESKE, J. C.; HUANG, E.; TOBACK, F. G. 2000. Regulation of renal epithelial cell affinity for calcium oxalate monohydrate crystals. Am. J. Physiol. 278: F130F137.

LIESKE, J.; COE, F. 1996. Urinary inhibitors and renal stone formation. En: Kidney Stones: Medical and Surgical Management. Coe, F.; Favus, M.; Pak C.; Parks, J.; Preminger, G. Lippincot-Raven, Philadelphia.

LIU, M.; RAGHEB, A.; ZELISKO, P.; BROOK, M. 2003. Preparation and applications of silicone emulsions using biopolymers. En: Elaïssari, Abdelhamid (Eds.), Biomedical Applications of Polymer Colloids, pp. 747-765, Mercel Dekker Inc.

LULICH, J. P.; OSBORNE, C. A.; BARTGES, J. W.; LEKCHAROENSUK, C. 2000. Canine lower urinary tract disorders. En: Ettinger, S. J.; Feldman, E. C. (eds) Textbook of Veterinary Internal Medicine-Diseases of Dog and Cat, 5ta edn., , Saunders, Philadelphia, pp 1747-1781.

LUND, E. M.; ARMSTRONG, P. J.; KIRK, C. A.; KOLAR, L. M.; KLAUSNER, J. S. 1999. Health status and population characteristics of dogs and cats examined at prívate veterinary practices in the United States. J. Am. Vet. Med. Assoc. 214: 1336-1341.

Marangella, M.; Futtero, M.; Bruno, M.; Linari, F. 1982. Hyperoxaluria in ideopathic calcium stone disease: further evidence of intestinal hyperabsorbtion of oxalate. Clin. Sci. 63: 381-385.

MARCHAND, J. E.; SANT, G. R.; KREAM, R. M. 1998. Increased expression of substance $\mathrm{P}$ receptor-encoding mRNA in bladder biopsies from patients with interstitial cystitis. Br. J. Urol. 81: 224-228.

MASSEY, L. K.; SUTTON, R. A. 1993. Modification of dietary oxalate and calcium reduces urinary oxalate in hyperoxaluric subjects with kidney stones. J. Am. Diet Assoc. 93: 13051307.-

MAZZALI, M.; KIPARI, T.; OPHASCHAROENSUK, V.; WESSON, J.; JOHNSON, R.; HUGHES, J. 2002. Osteopontin - a molecule for all seasons. QJM 95: 3-13.

MONJE, P.; BARAN, E. 2002. Characterization of calcium oxalates generated as biominerals in cacti. Plant Physiol. 128: 707-713. 
MONNERON, M. C.; GILlBERG, P. G.; OHMAN, B.; ALBERTS, P. 2000. In vitro alpha-adrenoceptor autoradiography of the urethra and urinary bladder of the female pig, cat, guinea-pig and rat. Scand. J. Urol. Nephrol. 34: 233-238.

NEIRA-CARRILLO, A.; KRISHNA PAI, R.; FERNANDEZ, M.S.; CARREÑO, E.; VÁSQUEZQUITRAL, P.; YAZDANI-PEDRAM, M., ARIAS, J.L. 2009. Synthesis and characterization of sulfonate polymethylsiloxane polymer as template for crystal growth of $\mathrm{CaCO}_{3}$. Colloid Polym. Sci. 287: 385-393.

NEIRA-CARRILLO， A.; VÁSQUEZ-QUITRAL， P.; YAZDANI-PEDRAM, M., ARIAS, J.L. 2010. Selective calcium oxalate crystallization induced by carboxylated monomethylitaconate grafted polymethylsiloxane. Liq. Cryst. \& Mol. Cryst. 521: 307-317.

NISHIURA, J. L.; MENDONCA, C. O. G.; SCHOR, N.; HEILBERG, I. P. 2002. Effect of calcium intake on urinary oxalate excretion in calcium stone forming subjects. Braz. J.

Med. Biol. Res. 35: 669-675.

PERTOVAARA, A.; KAUPPILA, T.; JYVÄSJÄRVI, E.; KALSO, E. 1991. Involvement of supraspinal and spinal segmental alpha-2-adrenergic mechanisms in the medetomidine-induced antinociception. Neuroscience 44: 705-714.

PRENEN, J. A.; BOER, P.; MEES, E. J. 1984. Absorption kinetics of oxalate from oxalate-rich food in man. Am. J. Clin. Nutr. 40: 1007-1010.

ROBERTSON W. G.; PEACOCK, M. 1980. The cause of idiopathic calcium stone disease: hypercalciuria or hyperoxaluria? Nephron 26:105. 266.

RUPNIAK N. M.; KRAMER, M. S. 1999. Discovery of the antidepressant and anti-emetic efficacy of substance $\mathrm{P}$ receptor (NK1) antagonists. Trends Pharmacol. Sci. 20: 485-490.

SANDS S. A.; STRONG, R.; CORBITT, J.; MORILAK, D. A.. 2000. Effects of acute restraint stress on tyrosine hydroxylase mRNA expression in locus coeruleus of Wistar and Wistar-Kyoto rats. Brain Res. Mol. Brain Res. 75: 1-7.

SCHWARZ, K. 1961. Separation of enol and keto tautomers of aromatic pyruvic acids by paper chromatography. Arch Biochem. Biophys. 92: 168-175.
SERAFINI-CESSI, F.; MALAGOLINI, N.; CAVALLONE, D. 2003. Tamm-Horsfall glycoprotein: biology and clinical relevance. Am. J. Kidney Dis. 42: 658-676.

SIENER, R.; HESSE, A. 1995. Influence of a mixed and a vegetarian diet on urinary magnesium excretion and concentration. Br. J. Nutr. 73: 783-790.

STAUFFER, J. K. 1977. Hyperoxaluria and calcium oxalate nephrolithiasis after jejunoileal bypass. Am. J. Clin. Nutr. 30: 64-71.

STEVENS C. W.; BRENNER, G. M. 1996. Spinal administration of adrenergic agents produces analgesia in amphibians. Eur. J. Pharmacol. 316: 205-210.

SUGAYA, K.; NISHIJIMA, S.; YAMADA, T.; MIYAZATO, M.; HATANO, T.; OGAWA, Y. 2002. Molecular analysis of adrenergic receptor genes and interleukin-4/interleukin-4 receptor genes in patients with interstitial cystitis. J. Urol. 168: 2668-2671.

TAKEI, K.; ITO, H.; MASAI, M.; KOTAKE, T. 1998. Oral calcium supplement decreases urinary oxalate excretion in subjects with enteric hyperoxaluria. Urol. Int. 61: 192-195.

TOMASZEWSKI, J.E.; LANDIS, J.; RUSSACK, V.; WILLIAMS, T.; WANG, L. P.; HARDY, C.; BRENSINGER, C.; MATTHEWS, Y. L.; ABELE, S. T.; KUSSEK, J. W. 2001. Biopsy features are associated with primary symptoms in interstitial cystitis: results from the interstitial cystitis database study. Urology 57: 67-81.

TSUJIHATA, M. 2008. Mechanism of calcium oxalate renal stone formation and renal tubular cell injury. Int $\mathrm{J}$ Urol 15: 115-120.

VALENTINO, R. J.; MISELIS R. R.; PAVCOVICH, L. A. 1999. Pontine regulation of pelvic viscera: pharmacological target for pelvic visceral dysfunctions. Trends Pharmacol. Sci. 20: 253-260.

VAN ROOIJEN, J.; VOSKAMP, A.; KAMERLING, J.; VLIEGENTHART, J. 1999. Glycosilation sites and site specific glycosilation in human Tamm-Horsfall glycoprotein. Glycobiology 9: 21-30.

VON UNRUH, G. E.; VOSS, S.; SAUERBRUCH, T. 2003.

Reference range for gastrointestinal oxalate absorption test. J. Urol. 169: 687-690.

WASSERTEIN, A. G. 1998. Nephrolithiasis: acute management and prevention. Dis. Mon. 44: 196-213. 
WEGNER, G. 2000. Functional Polymers. Acta Materialia 48: 253-262.

WELK, K., BUFFINGTON, C. A. 2003. Effects of Interstitial Cystitis on Central Neuropeptide and Receptor Immunoreactivity in Cats. Columbus, Department of Human Anatomy. Ohio State University, p 31. 